International Journal of Pure and Applied Mathematics

Volume 103 No. 3 2015, 421-428

ISSN: 1311-8080 (printed version); ISSN: 1314-3395 (on-line version)

url: http://www.ijpam.eu

doi: http://dx.doi.org/10.12732/ijpam.v103i3.4

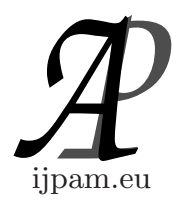

\title{
ON DOUBLE CONVERGENCE IN MEASURE
}

\author{
A. Gökhan \\ Department of Secondary Science \\ and Mathematics Education \\ Firat University \\ 23119 Elazı̆̆, TURKEY
}

\begin{abstract}
In this paper, we define some modes of convergence in the Pringsheim's sense for a double sequence $\left(f_{m n}\right)$ of measurable functions. We also give the relations between modes of these convergences.
\end{abstract}

AMS Subject Classification: 40B, 60F

Key Words: double sequence, double limit, convergence in measure

It is quite common in analysis, both for theoretical an practical purposes, to form infinite sequences of functions and to try to understand the limiting behavior of such sequences. Thus it is also quite meaningful to discuss convergence concepts. There are several possible modes of convergence for sequences of functions. The most important of these are the notions of pointwise and uniform convergence, but these convergence modes are too restrictive. In many cases, some of the function sequences do not convergence for every element in the do-

Received: March 26, 2015

(c) 2015 Academic Publications, Ltd. url: www.acadpubl.eu 
main. Therefore, the convergence modes in the weaker sense has been define; for example, almost everywhere convergence and convergence in measure and so on. In this part, we will summarize the knowledge given in some article. Krız and Stepan [6] show the conditions under which the almost everywhere convergence and the convergence in measure coincide. Komisarski [5] defined pointwise I-convergence and I-convergence in measure of sequences of measurable functions defined on a measure space with finite measure. Futhermore, He gived the relationship between these two convergences. Djurcic and Kocinac [2] proved that some classes of sequences of measurable functions satisfy certain selection principles related to special modes of convergence.

The notion of convergence for double sequences was first introduced by Pringsheim [8]. A double sequence $x=\left(x_{m n}\right)_{m, n=1}^{\infty}$ is said to be convergent in the Pringsheim's sense if for every $\varepsilon>0$ there exists $N \in \mathbb{N}$ such that $\left|x_{m n}-L\right|<\varepsilon$ whenever $j, k \geq N, L$ is called the Pringsheim limit ( or double limit) of $x$ and denoted by $P-\lim x_{m n}=L$.

Double sequences that the elements are functions are defined in the same way as single sequences of real-valued functions.

Throughout this note $(\Omega, \Gamma, \mu)$, or shortly $\Omega$, denotes a measure space with a complete measure $\mu: \Gamma \rightarrow \mathbb{R}$ (and $\Gamma$ is a $\sigma$-algebra of subsets of $\Omega$ measurable with respect to $\mu)$. $E$ is always an element in $\Gamma$ such that $\mu(E)<\infty$ [4], [9], [10].

A function $f: E \rightarrow \mathbb{R}$ is a measurable function if for each $c \in \mathbb{R}$,

$$
\{x \in E: f(x)>c\} \in \Gamma
$$

or ,equivalently, $f^{-1}(B) \in \Gamma$ for each $B \in B_{\mathbb{R}}$, where $B_{\mathbb{R}}$ is a $\sigma$-algebra of Borel sets in $\mathbb{R}$.

It is well known, in probability and statistics, a probability measure is a measure with total measure one, i.e. $\mu(\Omega)=1$. A probability space is a measure space with a probability measure. A random variable is a measurable real function whose domain is the probability space [1], [3], [7]

Throughout this paper, as we mentioned in Introduction, $(\Omega, \Gamma, \mu)$ will be always a measure space, and $E$ is an element in $\Gamma$ such that $\mu(E)<\infty$.

In this paper, we deal with some modes of convergence for a double sequence $\left(f_{m n}\right)$ of measurable functions on a set $E$.

As real-valued functions, the convergence of a double sequence of measurable functions $\left(f_{m n}\right)$ is defined as follows:

For every $x_{0} \in E,\left(f_{m n}\left(x_{0}\right)\right)$ converges to $f\left(x_{0}\right)$, i.e. $P-\lim _{m, n \rightarrow \infty} f_{m n}\left(x_{0}\right)=$ $f\left(x_{0}\right)$. Then we say that $\left(f_{m n}\right)$ converges (pointwise) everywhere on $E$. Now, we begin with the following definition being weaker than pointwise convergence. 
Definition 1. Let $\left(f_{m n}\right)$ be a double sequence of measurable functions on a set $E$, and let $f$ be a measurable function on $E$. Then $\left(f_{m n}\right)$ is said to converge almost everywhere, shortly a.e., to $f$ iff there exists a null set $M$ such that $\forall x \in E-M: P-\lim _{m, n \rightarrow \infty} f_{m n}(x)=f(x)$ finite.

This definition can be expressed as follows:

$$
\mu\left\{x \in E: P-\lim _{m, n \rightarrow \infty} f_{m n}(x) \neq f(x)\right\}=0
$$

We denote this symbolically by writting

$$
\text { a.e.P }-\lim _{m, n \rightarrow \infty} f_{m n}(x)=f(x) \text { or } f_{m n} \stackrel{P-a . e .}{\rightarrow} f \text { on } E \text {. }
$$

In measure theory, one talks about a.e. convergence of a sequence of measurable functions defined on a measure space. That means pointwise a.e. convergence .

Example 2. Let $\Omega=[0,1]$, we define $f_{m n}(x)$ by

$$
f_{m n}(x)= \begin{cases}1, & x \leq \frac{1}{m n} \\ 0, & x>\frac{1}{m n}\end{cases}
$$

and for every pair a,b $(0 \leq a, b \leq 1)$, we will require that $\mu(a \leq x \leq b)=b-a$.

This sequence $\left(f_{m n}(x)\right)$ converges a.e. to $f(x)=0$ on $\Omega$. For every $x \neq 0$ and every $\varepsilon>0$, there is an $k(\varepsilon)$ sufficiently large that for $m, n>k(\varepsilon)$,

$$
\left|f_{m n}(x)-f(x)\right|<\varepsilon .
$$

Thus, for $x \neq 0, P-\lim _{m, n \rightarrow \infty} f_{m n}(x)=f(x)$, and

$$
\mu\left\{x \in E: P-\lim _{m, n \rightarrow \infty} f_{m n}(x)=f(x)\right\}=\mu\{\Omega-\{0\}\}=1,
$$

i.e. a.e.P $-\lim _{m, n \rightarrow \infty} f_{m n}(x)=0$ on $\Omega$.

Definition 3. Let $\left(f_{m n}\right)$ be a double sequence of measurable functions on a set $E$, and let $f$ be a measurable function on $E$. Then the double sequence $\left(f_{m n}\right)$ is said to converge in measure on $E$ to $f$ iff for every $\varepsilon>0$, we have

$$
\lim _{m, n \rightarrow \infty} \mu\left\{x \in E:\left|f_{m n}(x)-f(x)\right| \geq \varepsilon\right\}=0 .
$$


We will indicate convergence in measure by writting

$$
\mu P-\lim _{m, n \rightarrow \infty} f_{m n}(x)=f(x) \text { or } f_{m n} \stackrel{P \mu}{\rightarrow} f \text { on } E .
$$

Notice that if $\mu(\Omega)=1$ then the convergence in measure is probabilty convergence on $E$.

Example 4. Let $\Omega, \mu$ and $\left(f_{m n}\right)$ be as given in Example 2. Then, we obtain $\mu P-\lim _{m, n \rightarrow \infty} f_{m n}(x)=f(x)=0$ on $\Omega$. Indeed, let

$$
A_{m n}(\varepsilon)=\left\{x \in \Omega:\left|f_{m n}(x)-f(x)\right|<\varepsilon\right\} .
$$

For $\varepsilon>1$, we have $A_{m n}(\varepsilon)=\Omega$ and $\mu\left\{A_{m n}(\varepsilon)\right\}=1$ for all $m, n$. For $0<\varepsilon \leq$ 1 ,we have $x \in A_{m n}(\varepsilon)$ if and only if $f_{m n}(x)=0$.Hence $\mu\left\{A_{m n}(\varepsilon)\right\}=1-\frac{1}{m n}$. Thus $\mu P-\lim _{m, n \rightarrow \infty} \mu\left\{A_{m n}(\varepsilon)\right\}=1$.

Theorem 5. Let $\left(f_{m n}\right)$ and $\left(g_{m n}\right)$ be a double sequence of measurable functions on a set $E$, and let $f$ and $g$ be two measurable function on $E$.

i) If $f_{m n} \stackrel{P \mu}{\rightarrow} f$ and $f_{m n} \stackrel{P \mu}{\rightarrow} g$ then $f=g$ a.e.

ii) If $f_{m n} \stackrel{P \mu}{\rightarrow} f$ and $g_{m n} \stackrel{P \mu}{\rightarrow} g$ then If $f_{m n} \pm g_{m n} \stackrel{P \mu}{\rightarrow} f \pm g$ on $E$.

Proof. It is easy to prove that.

Theorem 6. If $\left(f_{m n}\right)$ is a double sequence of measurable functions which converges in measure to $f$ on $E$, then there is a subsequence $\left(f_{m_{k} n_{k}}\right)$ which converges to $f$ a.e..

Proof. Choose $m_{1}, n_{1} \in \mathbb{N}$ such that

$$
\mu\left\{x \in E:\left|f_{m_{1} n_{1}}(x)-f(x)\right| \geq 1\right\}<\frac{1}{2} .
$$

Suppose that $n_{1}, n_{2} \ldots, n_{k}$ and $m_{1}, m_{2}, \ldots, m_{l}$ have been chosen. Then pick $n_{k+1}$ and $m_{l+1}$ such that $n_{k+1}>n_{k}$ and $m_{l+1}>m_{l}$ and

$$
\mu\left\{x \in E:\left|f_{m_{l+1}, n_{k+1}}(x)-f(x)\right| \geq \frac{1}{k+l+1}\right\}<\frac{1}{2^{k+l+1}} .
$$

Let

$$
A_{j}=\bigcup_{k, l=j}^{\infty}\left\{x \in E:\left|f_{m_{l}, n_{k}}(x)-f(x)\right| \geq \frac{1}{k+l}\right\}
$$


for each $j \in \mathbb{N}$. Clearly we have $A_{1} \supset A_{2} \supset A_{3} \supset \ldots$. Next let $B=\bigcap_{j=1}^{\infty} A_{j}$. Since

$$
\mu\left(A_{1}\right)<\sum_{k, l=1}^{\infty} \frac{1}{2^{k+l}}<\infty .
$$

It follows that

$$
\mu(B)=\lim _{j \rightarrow \infty} \mu\left(A_{j}\right) \leq \lim _{j \rightarrow \infty} \sum_{k, l=j}^{\infty} \frac{1}{2^{k+l}}=0,
$$

that is, $\mu(B)=0$. Next, let

$$
x \in E-B=\bigcup_{j=1}^{\infty}\left(E-A_{j}\right) .
$$

Then there is a $j_{x}$ such that

$$
x \in E-A_{j_{x}}={\stackrel{\bigcap}{h}, j_{x}}_{j_{x}}\left\{t \in E:\left|f_{m_{l}, n_{k}}(t)-f(t)\right|<\frac{1}{k+l}\right\} .
$$

Given $\varepsilon>0$, choose $k_{0}, l_{0}$ such that $k_{0}, l_{0} \geq j_{x}$ and $\frac{1}{k_{0}+l_{0}} \leq \varepsilon$. Then $k \geq k_{0}$ and $l \geq l_{0}$ implies that

$$
\left|f_{m_{l}, n_{k}}(x)-f(x)\right|<\frac{1}{k+l} \leq \varepsilon
$$

and this proves that $f_{m_{l}, n_{k}}(x) \rightarrow f(x)$ for all $x \in E-B$. The proof is finished.

Definition 7. Let $\left(f_{m n}\right)$ be a double sequence of measurable functions on a set $E$, and let $f$ be a measurable function on $E$. We say that $\left(f_{m n}\right)$ converges almost uniformly to $f$ if for every $\varepsilon>0$ there is a measurable set $E$ such that $\mu(E)<\varepsilon$ and $f_{m n} \rightarrow f$ uniformly on $E^{c}$. We will indicate convergence in almost uniformly by writing $f_{m n} \stackrel{P-a . u .}{\rightarrow} f$ on $E$.

Theorem 8. If $\left(f_{m n}\right)$ converges to $f$ a.u. then $\left(f_{m n}\right)$ converges to $f$ a.e. ( without assumption $\mu(F)<\infty$ ).

Proof. Let $E_{m n} \subseteq \Omega$ be a measurable set with the property that $\mu\left(E_{m n}\right)<$ $\frac{1}{2^{m+n}}$ and $f_{m n} \rightarrow f$ uniformly on $E_{m n}^{c}$. Let $F_{k l}=\bigcup_{m=k, n=l}^{\infty} E_{m n}$ and let $F=$ $\bigcap_{k, l=1}^{\infty} F_{k l}$. By countable subaddivity of measure, $\mu\left(F_{k l}\right) \leq \sum_{m=k, n=l}^{\infty} \frac{1}{2^{m+n}}=\frac{1}{2^{k+l-2}}$ 
and thus by continuity of measure from above we have $\mu(F)=0$. Moreover $F^{c}=\bigcup_{k, l=1}^{\infty} \bigcap_{m=k, n=l}^{\infty} E_{m n}^{c}$; since $f_{m n}$ converges uniformly and therefore pointwise to $f$ on each $E_{m n}^{c}$ it follows that $f_{m n}$ converges pointwise on $F^{c}$, i.e. $f_{m n} \stackrel{P-a . e .}{\rightarrow} f$ on $\mathrm{F}$.

Theorem 9. Assume that $\mu(E)<\infty$ and let $\left(f_{m n}\right)$ be a double sequence of measurable functions on a set $E$ and let $f$ be a measurable function on $E$ such that $f_{m n} \stackrel{P-a . e .}{\rightarrow} f$. Then $f_{m n}$ converges to $f$ almost uniformly .

Proof. Possibly adjusting on a set of measure 0, we may assume that $f_{m n}$ converges to $f$ everywhere on $E$. Consider the sets

$$
E_{k l}(m, n)=\bigcup_{p=k, q=l}^{\infty}\left\{x \in E:\left|f_{p q}(x)-f(x)\right| \geq \frac{1}{m+n}\right\} .
$$

The assumption that $f_{m n}$ converges pointwise to $f$ implies that $\bigcap_{k, l=1}^{\infty} E_{k l}(m, n)$ is empty for each fixed $m$ and $n$, so since $\mu(E)<\infty$ we may apply continuity from above to deduce that $\mu\left(E_{k l}(m, n)\right) \rightarrow 0$ as $k, l \rightarrow \infty$. Now, fix $\varepsilon>0$ and choose $m, n \in \mathbb{N}$. We may choose $k_{m}$ and $l_{n}$ so that $\mu\left(E_{k_{m}, l_{n}}(m, n)\right)<\varepsilon \frac{1}{2^{m+n}}$. The set we need is $E=\bigcup_{m, n=1}^{\infty} E_{k_{m}, l_{n}}(m, n)$ which by countable subadditivity has $\mu(E)<\varepsilon \sum_{m, n=1}^{\infty} \frac{1}{2^{m+n}}=\varepsilon$. If $x \in E^{c}$ then $x \in\left[E_{k_{m}, l_{n}}(m, n)\right]^{c}$ for every $m, n$, which means that $\left|f_{m n}(x)-f(x)\right|<\frac{1}{m+n}$ for every $m>k_{m}$ and $n>l_{n}$. By definition, this means $f_{m n}$ converges uniformly to $f$.

Corollary 10. If $\mu(E)<\infty$ and let $f_{m n}, f: E \rightarrow \mathbb{R}$ be a measurable functions for each $m, n \in \mathbb{N}$ on $E$, then the following holds: a.e. double convergence $\Leftrightarrow$ a.u. double convergence.

Theorem 11. Let $\left(f_{m n}\right)$ be a double sequence of measurable functions on a set $E$, and let $f$ be a measurable function on $E$. If $\left(f_{m n}\right)$ converges to $f$ almost uniformly then $\left(f_{m n}\right)$ converges to $f$ in measure.

Proof. For each $m, n \in \mathbb{N}$,let $E_{m n}$ be a measurable set such that $\mu\left(E_{m n}\right)<$ $\frac{1}{m+n}$ and $f_{m n} \rightarrow f$ uniformly on $E_{m n}^{c}$. The statement that $f_{m n} \rightarrow f$ uniformly means that for every $\varepsilon>0$ there exists $N_{m n}$ such that $\left|f_{k l}(x)-f(x)\right|<\varepsilon$ whenever $k, l>N_{m n}$ for any $x \in E_{m n}^{c}$. This means that for $k, l>N_{m n}$, 
$\left\{x \in E:\left|f_{k l}(x)-f(x)\right| \geq \varepsilon\right\} \subseteq E_{m n}$ and hence

$$
\mu\left\{x \in E:\left|f_{k l}(x)-f(x)\right| \geq \varepsilon\right\}<\frac{1}{m+n} .
$$

Passing to the limit as $m, n \rightarrow \infty$, we conclude that

$$
\mu\left\{x \in E:\left|f_{k l}(x)-f(x)\right| \geq \varepsilon\right\} \rightarrow 0
$$

as $k, l \rightarrow \infty$.

Corollary 12. Let be $\mu(E)<\infty$ and $f_{m n}, f: E \rightarrow \mathbb{R}$ for each $m, n \in \mathbb{N}$ be measurable functions on a set $E$. If $f_{m n} \stackrel{P-a . e .}{\rightarrow} f$ (or equivalently $f_{m n} \stackrel{P-a . u .}{\rightarrow} f$ ) on $E$, then $f_{m n} \stackrel{P \mu}{\rightarrow} f$ on $E$.

We note that the converse is not true. There are sequences of functions that converge in measure and do not converge almost everywhere.

For example, consider the sequence $f_{m n}:[0,1] \rightarrow \mathbb{R}$ defined by

$$
f_{m n}(x)=\left\{\begin{array}{cc}
1, & \frac{j}{2^{k}} \leq x \leq \frac{j+1}{2^{k}}, n=2^{k}+j, 0 \leq j<2^{k}, m=1,2, \ldots \\
0, & \text { otherwise }
\end{array}\right.
$$

where $\mu(a \leq x \leq b)=b-a$. Define the $f(x)$ (real-valued) by $f(x)=0$. Then for $\varepsilon>1$, trivially, $\mu\left\{x \in[0,1]:\left|f_{m n}(x)\right| \geq \varepsilon\right\}=0$ and for $0<\varepsilon \leq 1$, $\mu\left\{x \in[0,1]:\left|f_{m n}(x)\right| \geq \varepsilon\right\} \leq \frac{1}{2^{k}} \rightarrow 0$ as $m, n \rightarrow \infty$, where $\mathrm{k}$ is the largest integer with the property that $2^{k} \leq n$. Thus $\left(f_{m n}\right)$ converges to 0 in measure.However $f_{m n}(x)$ does not converge almost everywhere to $f(x)$. To see this , observe that for any $x \in[0,1]$ and any positive integer $N$, there are two $m, n>N$, such that $f_{m n}(x)=1$. In fact $f_{m n}(x)$ converges to $f(x)$ at no point of $[0,1]$.

In the above examples, the double limit $f(x)$ was the constant function $f(x)=0$. There is no loss in generality in using a constant function, since if $f(x)$ is not constant, and $f_{m n}(x) \rightarrow f(x)$, we can define $g_{m n}(x)=f_{m n}(x)-f(x)$, and then $g_{m n}(x) \rightarrow 0$.

\section{References}

[1] K.L. Chung, A Course in Probabilty Theory, Academic Press (1968). 
[2] D. Djurcic, L.D.R. Kocinac, A note on convergence in measure and selection principles, Filomat, 26, No. 3 (2012), 473-477.

[3] B. Harris, Theory of Probability, Addison-Wesley Publishing (1966).

[4] G. Klambauer, Real Analysis, American Elsevier Pub. Co. (1973).

[5] A. Komisarski, Pointwise I-convergence and I-convergence in measure of sequences of functions, J. Math. Anal. Appl., 340 (2008), 770-779.

[6] P. Krız, J. Stepan, A note on almost sure convergence and convergence in measure,

it Comment. Math. Univ. Carolin., 55, No. 1 (2014), 29-40.

[7] A. Papoulis, The Concept of a Random Variable, Ch. 4 in Probability, Random Variables, and Stochastic Processes, 2nd ed. New York: McGrawHill, pp. 83-115 (1984).

[8] A. Pringsheim, Zur Ttheorie der zweifach unendlichen Zahlenfolgen, Math. Ann., 53 (1900), 289-321.

[9] H.L. Royden, Real Analysis, Staford University Macmillan Publishing Co. (1968).

[10] R.L. Wheeden, A. Zygmund, Measure and Integral, Marcel Dekker, New York (1977). 